\title{
AN EQUALITY FOR 2-SIDED SURFACES WITH A FINITE NUMBER OF WILD POINTS
}

\author{
BY \\ MICHAEL D. TAYLOR AND HARVEY ROSEN
}

\begin{abstract}
Let $S$ be a 2-sided surface in a 3-manifold that is wild from one side $U$ at just $m$ points. It is shown that the minimal genus possible for all members of a sequence of surfaces in $U$ converging to $S$ (where these surfaces each separate the same point from $S$ in $U \cup S$ ) is equal to the sum of the genus of $S$ and a certain multiple of the sum of $m$ special topological invariants associated with the wild points. In this equality, the sum of these invariants is multiplied by just one of the numbers 0,1 , or 2, dependent upon the genus and orientability class of $S$ and the value of $m$. As an application, an upper bound is given for the number of nonpiercing points that a 2-sided surface has with respect to one side.
\end{abstract}

1. Introduction and notation. Let $S$ be a 2 -sided surface of genus $g(S)$ embedded in a 3-manifold $M^{3}$. There is an open connected neighborhood $W$ of $S$ such that $W-S$ consists of exactly two components, $U$ and $V$. We define $g(S, U)$ to be $n$ if there exist a point $x$ and a sequence of surfaces $S_{1}, S_{2}, \ldots$ such that

(1) the genus of each $S_{k}$ is $n$,

(2) each $S_{k}$ separates $x$ from $S$ in $\mathrm{Cl}(U)$,

(3) $S=\lim S_{k}$, and

(4) conditions (1)-(3) cannot be satisfied for any integer smaller than $n$.

If no such integer $n$ exists we define $g(S, U)$ to be $\infty$. This concept coincides with that of limiting genus of $U$ at $S$ or limiting genus of $U$ which is used by Daverman in [7] and [8]. In [13] it is shown that if $S$ can be locally peripherally collared from $U$ and $S$ is a 2-sphere in $S^{3}$ and $g(S, U)$ is positive, then $S$ is wild from $U$ at $g(S, U)$ points at most. More generally, in [7] it is proven that if $S$ can be locally peripherally collared from $U, g(S, U)$ is positive, and $S$ is an orientable surface which separates $M^{3}$, then it follows that $g(S, U)-g(S)$ is an upper bound for the number of points at which $S$ is wild from $U$. The equality developed in this paper sheds some light on why the above inequalities hold and is of interest in its own right. The authors are indebted to the referee for his constructive suggestions.

Let us suppose that either $S$ is wild from $U$ at $p_{1}, \ldots, p_{m}$ and nowhere else or else $S$ is tame from $U(m=0)$. To each point $q$ of $S$ we can attach a nonnegative integer $g(q, U)$ which is a topological invariant of $\mathrm{Cl}(U)$. This has the property

Presented to the Society, July 22, 1969; received by the editors May 21, 1971.

AMS 1970 subject classifications. Primary 55A30, 57A10.

Key words and phrases. 2-sided surfaces in 3-manifolds, surfaces with finitely many wild points, convergent sequence of surfaces, limiting genus, local enveloping genus, number of nonpiercing points of surfaces. 
that $g(q, U)$ is positive only when $q$ is one of the $p_{k}$ and otherwise is 0 . Our main result is that

$$
\begin{aligned}
(S, U) & =g(S) & & \text { if } m=0, \\
& =0 & & \text { if } m=1 \text { and } g(S)=0, \\
& =g(S)+\sum_{k=1}^{m} g\left(p_{k}, U\right) & & \text { if } S \text { is orientable and either } m>1 \text { or } g(S) \neq 0, \\
& =g(S)+2 \sum_{k=1}^{m} g\left(p_{k}, U\right) & & \text { if } m \neq 0 \text { and } S \text { is nonorientable. }
\end{aligned}
$$

Further we are able to show that we can always find arcs $A_{1}, \ldots, A_{m}$ such that each $A_{k}$ has $p_{k}$ for an endpoint and $g\left(p_{k}, U\right)=\operatorname{LEG}\left(A_{k}, p_{k}\right)$ (where LEG $\left(A_{k}, p_{k}\right)$ is the local enveloping genus of $A_{k}$ at $p_{k}$ ). If $S$ is locally tame from $V$ at $p_{k}$, then we can take $A_{k}$ to be any arc on $S$ which has $p_{k}$ as an endpoint.

Before attempting to prove anything, however, let us discuss some terms and notation.

It is understood that a manifold and a manifold-with-boundary are both considered to be connected. A manifold may or may not be compact, but it does not have any boundary points. A surface is a compact 2-manifold. Our results are still true if $M^{3}$ is a manifold-with-boundary, but then we should stipulate that $S$ lies in the interior of $M^{3}$.

Let $K$ be a subset of an $n$-manifold $M^{n}$. Then $K$ is locally polyhedral at a point $p$ if there is a neighborhood $N$ of $p$ such that $K \cap \mathrm{Cl}(N)$ is a polyhedron in $M^{n}$. We say that $K$ is locally polyhedral if it is locally polyhedral at each point.

Let $S, M^{3}, U$, and $V$ be as defined earlier. We shall call $U$ and $V$ the (nonunique) sides of $S$. If $p$ is a point of $S$, then we say that $S$ is locally tame from $U$ at $p$ if $\mathrm{Cl}(U)$ is a 3-manifold-with-boundary at $p$. If $\mathrm{Cl}(U)$ is a 3-manifold-with-boundary at every point of $S$, then $S$ is tame from $U$. If $p$ is a point of $S$ where $S$ is not locally tame from $U$, then $S$ is wild from $U$ at $p$.

We shall also use the following notations:

$a b$-an arc with $a$ and $b$ as endpoints,

Bd-the boundary of a manifold-with-boundary,

Int-the interior of a manifold-with-boundary,

$\mathrm{Cl}$-closure,

$d$-metric (used for giving the distance between sets as well as points),

diam-diameter,

$I-[0,1]$,

$N(K, \varepsilon)$-the $\varepsilon$-neighborhood of a set $K$,

$g(F)$ - the genus of a surface $F$ or of a surface-with-boundary,

ch $(F)$-the Euler-Poincaré characteristic of $F$.

Let $S, M^{3}, W, U$ and $V$ be as defined before. We wish to introduce a new term.

Definition. Suppose that $S$ is wild from $U$ at a finite number of points at most.

Let $p$ be a point of $S$ and consider this statement which we label $G(p, m)$ : 
For every positive number $\varepsilon$ there are a disc with $m$ handles $D$ and a disc $D^{\prime}$ such that $p \in \operatorname{Int}\left(D^{\prime}\right) \subset S, D \cap S=\operatorname{Bd}(D)=\operatorname{Bd}\left(D^{\prime}\right)$, Int $(D) \subset U$, diam $\left(D \cup D^{\prime}\right)$ $<\varepsilon$. If $G(p, m)$ is true and $G(p, m-1)$ is not true, then we define $g(p, U)$ to be $m$. If $G(p, m)$ is false for $m=0,1,2, \ldots$, we define $g(p, U)$ to be $\infty$. Notice that this is in some sense a generalization of Burgess' concept of local spanning in [4]. The surface $S$ can be locally spanned from $U$ at $p$ if and only if $g(p, U)=0$. If $g(p, U)>0$, then $S$ is wild from $U$ at $p$. This definition can of course be immediately extended to the situation where $S$ can be locally peripherally collared from $U$. We wish to use this concept of $g(p, U)$ in proving our equalities and shall show in the last section that it is equivalent to the idea of local enveloping genus that we referred to earlier.

Letting $S, M^{3}, W, U$, and $V$ be as defined earlier, we establish our equalities by breaking them up into inequalities which we establish separately. The following lemma gives us an upper bound for $g(S, U)$.

LEMMA 1. Let us suppose that $S$ is wild from $U$ at the points $p_{1}, \ldots, p_{m}$ and nowhere else (this proof is also valid if $S$ is tame from $U$, i.e., if $m=0$ ). Then

$$
\begin{array}{ll}
g(S, U) \leqq g(S)+\sum g\left(p_{k}, U\right) & \text { if } S \text { is orientable, } \\
g(S, U) \leqq g(S)+2 \sum g\left(p_{k}, U\right) & \text { if } S \text { is nonorientable. }
\end{array}
$$

Proof. We may suppose that each $g\left(p_{k}, U\right)$ is finite since otherwise the inequality is trivial.

Choose a point $x$ of $U$ not lying in an arbitrary $\varepsilon$-neighborhood $N(S, \varepsilon)$. There exist pairwise disjoint discs $D_{1}, \ldots, D_{m}$ and pairwise disjoint discs with handles $H_{1}, \ldots, H_{m}$ such that, for $i=1, \ldots, m, p_{i} \in \operatorname{Int}\left(D_{i}\right) \subset S, H_{i} \cap S=\operatorname{Bd}\left(H_{i}\right)=\operatorname{Bd}\left(D_{i}\right)$, Int $\left(H_{i}\right) \subset U, H_{i}$ has $g\left(p_{i}, U\right)$ handles, and diam $\left(H_{i} \cup D_{i}\right)<\varepsilon$.

We may suppose that each surface $H_{k} \cup D_{k}$ is contained is some open 3-cell and that $U_{k}$ is the complementary domain of $H_{k} \cup D_{k}$ which lies in that open 3-cell. By [1], we may assume that $M=(S \cup U)-\bigcup_{k=1}^{m}\left(U_{k} \cup\right.$ Int $\left.D_{k}\right)$ is a 3-manifoldwith-boundary, and $\mathrm{Bd} M=\left(S-\bigcup_{k=1}^{m} D_{k}\right) \cup\left(\bigcup_{k=1}^{m} H_{k}\right)$.

Let $R$ be a regular neighborhood of $\mathrm{Bd} M$ in $M$ such that $R \subset N(S, \varepsilon)$. Call $F$ the component of $\mathrm{Bd}(R)$ that lies in $U$. By construction, $F$ separates $x$ from $S$ and has genus equal to $g(S)+\sum g\left(p_{k}, U\right)$ if $S$ is orientable or $g(S)+2 \sum g\left(p_{k}, U\right)$ if $S$ is nonorientable. From the existence of such a surface $F$ in any $\varepsilon$-neighborhood of $S$, the conclusion of the lemma follows.

2. Some intermediate lemmas. Before we can prove the inequality in the opposite direction (except, as we shall see, for the case when $S$ is a 2-sphere wild from $U$ at a single point), we shall need to establish some lemmas.

Lemma 2. Let $S$ be a surface or a surface-with-boundary in a 3-manifold $M^{3}$ and let $O$ be a neighborhood of $S$. Then there are a neighborhood $N$ of $S$ and a homotopy $h: N \times I \rightarrow O$ such that $S \subset N \subset O, h_{0}=$ the identity map, and $h_{1}$ is a retract map of $N$ onto $S$. 
A proof of this can be found on pp. 97, 121, and 122 of [10].

The next proof amounts to a generalization of certain constructions used by Burgess in [5], but the details are sufficiently involved to make it worth going over.

Lemma 3. Suppose that $S$ is a 2-manifold in a 3-manifold $M^{3}$ such that $U$ is a complementary domain of $S$ and $p$ is a point of $S$. Let $\delta, D$, and $A$ be a positive real number, disc, and annulus respectively such that $p \in \operatorname{Int}(D) \subset S, A \cap S=\operatorname{Bd}(D)$ =one of the boundary components of $A, A-S \subset U, A-S$ is locally polyhedral, and $\operatorname{diam}(A \cup D)<\delta$.

If $\delta$ is sufficiently small, then given $\delta, D$, and $A$ as described above, there exists a positive real number $\sigma$ such that the following holds:

If $F$ is a locally polyhedral 2-manifold in $U \cap N(S, \sigma)$ which intersects $A$ and is in general position with respect to $A$, then there exists a component $C$ of $F-A$ such that diam $(C)<7 \delta$.

Proof. Choose a disc $D^{\prime}$ in $S$ so that $p$ is a point in the interior of $D^{\prime}$. There is a positive real number $\varepsilon$ such that the intersection of $N(p, 5 \varepsilon)$ and $S$ is contained in the interior of $D^{\prime}$. Suppose that $\delta, A$, and $D$ are as described in the hypothesis and $\delta<\varepsilon$. Clearly $D$ is a subset of Int $\left(D^{\prime}\right)$. Let $J$ be the name of that boundary component of $A$ which lies in $U$. By using Lemma 2 and looking at collections of 3-cells which contain $D^{\prime}$ in the unions of their interiors, we see that there are a positive real number $\sigma^{\prime}$, a neighborhood $N$ of $D^{\prime}$, and a homotopy $h_{t}$ of $N$ in $M^{3}$ such that $h_{0}=$ the identity map on $N, h_{1}$ is a retract map of $N$ onto $D^{\prime}, h_{t}(N)$ does not intersect $J$ for $0 \leqq t \leqq 1, \sigma^{\prime}<\varepsilon, N\left(D^{\prime}, \sigma^{\prime}\right)$ is a subset of $N$, and if $x$ and $y$ are points of $N$ and the distance from $x$ to $y$ is less than $\sigma^{\prime}$, then there exists an arc $x y$ in $N$ such that diam $(x y)<\delta$.

Then we can find a positive real number $\sigma$ and a subannulus $A^{\prime}$ of $A$ such that $\sigma<\sigma^{\prime}, \mathrm{Bd}(D)$ is one of the components of $\mathrm{Bd}\left(A^{\prime}\right)$, and $N\left(D^{\prime}, \sigma\right) \cap A \subset A^{\prime} \subset N\left(D^{\prime}, \sigma^{\prime}\right)$.

Now let $F$ be as in the hypothesis. It follows that $F$ intersects $A$ only at points of $A^{\prime}$. By using the methods of Wilder [14, p. 66] and "walking over" $D$ and "up the side" of $A^{\prime}$, we may construct an arc $p a$ such that $a$ is a point in $F$, Int $(p a)$ is contained in $U-A, p a$ is contained in $N\left(S, \sigma^{\prime}\right)$, and diam $(p a)<\delta$.

It follows that $p a$ is contained in $N\left(D^{\prime}, \sigma^{\prime}\right)$. Let $C=$ the component of $F-A$ containing $a$. Now suppose that $\operatorname{diam}(C)$ is greater than or equal to $7 \delta$. Then there is an arc $a b$ in $C$ such that the distance from $a$ to $b=3 \delta$ and for $z$ in Int $(a b)$ the distance from $a$ to $z$ is less than $3 \delta$. Since $a b$ is in $N(S, \sigma)$, there must be a point $c$ in $S$ which is a distance less than $\sigma$ from $b$. It can be easily shown that $b$ is in $N$ and $c$ is in Int $\left(D^{\prime}\right)$ and hence also in $N$. By the way we constructed $N$, there exists an $\operatorname{arc} b c$ in $N$ such that diam $(b c)<\delta$. It follows, in addition, that $a b$ is contained in $N$ and that $b c$ does not intersect $A$ or $D$. Let $c p$ be an arc in $N$ such that Int $(c p)$ does not intersect $A$ or $D$, and let $J^{\prime}$ be the continuous image of a simple closed curve which can be obtained by considering the union of $p a, a b, b c$ and $c p$. Since $J^{\prime}$ is contained in $N$, it can be homotopied into $D^{\prime}$ and from there into $U$ without 
intersecting $J$. On the other hand, $J^{\prime}$ intersects the disc $D \cup A$ at the point $p$ only and there it pierces it. But this shows that the mod 2 linking number of $J^{\prime}$ with $J$, calculated with respect to the fixed surface-with-boundary $D \cup A$, must be both 0 and 1 , an impossibility. Therefore $\operatorname{diam}(C)<7 \delta$.

Lemma 4. Let $S, M^{3}, W, U$, and $V$ be as in the Introduction and let $S$ be wild from $U$ at a finite number of points at most. Then there is an $\varepsilon>0$ such that if $F$ is a surface in $U$ with the property that $F$ separates $S$ in $\mathrm{Cl}(U)$ from the set of points of $U$ which are more than a distance $\varepsilon$ from $S$, then $F$ is of the same orientability class as $S$.

Proof. Suppose that $S$ is orientable. By Theorem 2 of [12], there exists a positive number $\varepsilon_{1}$ such that $N\left(S, \varepsilon_{1}\right)$ can be embedded in $S^{3}$. Let $\varepsilon=\varepsilon_{1} / 2$. This automatically ensures that $F$ can be embedded in $S^{3}$ and hence is orientable.

Now suppose that $S$ is nonorientable. We can find two Moebius bands $M$ and $M^{*}$ on $S$ such that neither one contains any of the points at which $S$ is wild from $U$ and $M \subset \operatorname{Int}\left(M^{*}\right)$. By Theorem 1 of [3], there is a homeomorphism $h$ such that $h:$ Int $\left(M^{*}\right) \times[0,2] \rightarrow \mathrm{Cl}(U), h(x, 0)=x$ for every $x \in \operatorname{Int}\left(M^{*}\right)$ and

$$
h\left(\operatorname{Int}\left(M^{*}\right) \times(0,2]\right) \subset U .
$$

Choose $\varepsilon$ so that $h(M \times 1)$ is a distance more than $\varepsilon$ from $S$. Let $F$ be as in the statement of the lemma. By [2] we may assume $F$ to be polyhedral. There is a positive number $\delta$ such that $F \cap h(M \times I) \subset h(M \times(\delta, 1))$. Since $M \times[\delta, 1]$ is locally tame in Int $\left(M^{*}\right) \times[0,2]$, by Theorem 9 of [1] we may assume that $h(M \times[\delta, 1])$ is polyhedral (we can adjust it by a very small homeomorphism of $M^{3}$ onto itself) and satisfies all the earlier requirements put on it. We may then further assume that $F$ and $h(\mathrm{Bd}(M) \times I)$ are in general position with respect to one another. It follows that $F \cap h(M \times I)$ consists of a finite number of polyhedral surfaces-with-boundary $F_{1}, \ldots, F_{n}$ such that for $k=1, \ldots, n, \mathrm{Bd}\left(F_{k}\right) \subset h(\mathrm{Bd}(M) \times I)$,

$$
\text { Int }\left(F_{k}\right) \subset h(\operatorname{Int}(M) \times I) \text {, }
$$

and $\bigcup_{k=1}^{n} F_{k}$ separates $h(M \times 0)$ and $h(M \times 1)$ in $h(M \times I)$.

From Lemma 4 of [9] we see that at least one of the $F_{k}$ must be nonorientable. Thus $F$ is nonorientable.

3. The main theorem. Since we have Lemma 1, all we have to do is find an appropriate lower bound for $g(S, U)$. Then in the next section we shall show how to replace $g(p, U)$ by the local enveloping genus of a suitable arc at an endpoint.

THEOREM 1. Let $S$ be a 2-sided surface in a 3-manifold $M^{3}$ and let $W$ be an open connected neighborhood of $S$ such that $W-S$ consists of two components, $U$ and $V$ (the two sides of $S$ ). We suppose that $S$ is wild from $U$ at the points $p_{1}, \ldots, p_{m}$ and nowhere else. Then

$$
g(S, U)=0 \text { if } g(S)=0 \text { and } m=1,
$$




$$
g(S, U)=g(S)+\sum_{k=1}^{m} g\left(p_{k}, U\right) \quad \text { if } S \text { is orientable and either } g(S) \neq 0 \text { or } m \neq 1
$$

and

$$
g(S, U)=g(S)+2 \sum_{k=1}^{m} g\left(p_{k}, U\right) \text { if } S \text { is nonorientable. }
$$

Prior to giving a proof of Theorem 1, we first offer a rough outline of how it goes. We essentially start with one collection (out of infinitely many collections) of $m$ annuli each sticking into $U$ except for a boundary component on $S$ circling a wild point of $S$, and we suppose we have a surface of genus $g(S, U)$ in $U$ which is sufficiently close to $S$, separates the boundary components of each annulus in the collection, and is in general position with respect to these annuli. We then surgically alter this surface until we ultimately obtain at the end of the operation a surface with genus no greater than the original surface and with all the special properties of the original one except that the new surface intersects each annulus nicely in exactly one simple closed curve provided, as is eventually shown, each annulus is small enough. This new surface is made up of two types of pieces: (1) $m$ small discs with handles, each cut out by an annulus, and (2) a large connected piece which is what is left when the small discs with handles are removed. The inequality is then established by showing that the sum of the genera of the small pieces must be no smaller than the sum (or twice the sum) of the local enveloping genera of the wild points of $S$ and that the genus of the large piece must be no smaller than the genus of $S$.

Proof of Theorem 1. Before getting into the thick of difficulties, we consider the case in which $S$ is a 2-sphere which is wild from $U$ at a single point. It follows from the fact that a neighborhood of $S$ may be embedded in $S^{3}$ [12] and from [6] and [1] that $g(S, U)=0$. So we may suppose that either $g(S) \neq 0$ or $m \geqq 2$.

Step 1. We need first of all to construct a number of objects which will be used in the proof. We suppose $W$ chosen so close to $S$ that $W$ can be homotopied onto $S$ and any point of $S$ will go onto itself (guaranteed by Lemma 2). For $i, j=1, \ldots, m$ and $k=1,2, \ldots$, we can find $\operatorname{discs} D_{i k}$ and annuli $A_{i k}$ such that $p_{i} \in \operatorname{Int}\left(D_{i k}\right) \subset S$; $\mathrm{Bd}\left(D_{i k}\right)=$ one of the boundary components of $A_{i k} ; A_{i k}-S \subset U ; J_{i k}$ is the name we give to the boundary component of $A_{i k}$ in $U$; $\operatorname{diam}\left(A_{i k} \cup D_{i k}\right)<\delta_{k}$, where $\delta_{k}$ is a positive number chosen sufficiently small to make Lemma 3 work for $D_{i k}$ and $A_{i k}$ $(i=1, \ldots, m)$ and such that $\delta_{k}<1 /\left(7 \times 2^{k}\right)$; and $\left(A_{i k} \cup D_{i k}\right) \cap\left(A_{j k} \cup D_{j k}\right)=\varnothing$ if $i \neq j$.

We then know that for every $k$ we can find $\sigma_{k}$ such that $0<\sigma_{k}<\delta_{k}$ and the conclusions of Lemma 3 hold for $\sigma_{k}$ with respect to $\delta_{k}$. By [2] we may suppose each $A_{i k}-S$ is locally polyhedral. For $k=1,2, \ldots$, let $G_{k}$ be a connected polyhedral 3-manifold-with-boundary in $U$ such that $G_{k}$ contains all points of $U$ which are a distance greater than or equal to $\sigma_{k} / 2, G_{k}$ contains $J_{1 k}, \ldots, J_{m k}$, and any surface $F$ in $U$ which separates $S$ from $G_{k}$ in $\mathrm{Cl}(U)$ must have the same orientability class as $S$ (Lemma 4$)$. 
Next for $k=1,2, \ldots$ and $i=1, \ldots, m$, we choose $A_{i k}^{\prime}$ such that $A_{i k}^{\prime}$ is a subannulus of $A_{i k}, \mathrm{Bd}\left(D_{i k}\right)=$ one of the boundary components of $A_{i k}^{\prime}$, and $A_{i k}^{\prime} \subset N\left(S, \sigma_{k}\right)$.

Now we describe a condition which will be useful in the rest of the proof. We say that a polyhedral surface $F$ in $U$ satisfies condition $\left(\mathrm{C}_{k}\right)$ if and only if

$F$ separates $S$ from $G_{k}$ in $\mathrm{Cl}(U)$,

$F$ is in general position with respect to each $A_{i k}$, and

$F \cap A_{i k} \subset \operatorname{Int}\left(A_{i k}^{\prime}\right)$ for $i=1, \ldots, m$.

Note that $F$ must have the same orientability class as $S$, that $F \cap A_{i k}$ must contain a component which is an essential simple closed curve in $A_{i k}$, and that $F$ is contained in $N\left(S, \sigma_{k}\right)$. Finally, for $k=1,2, \ldots$, we choose $M_{k}$ to be a polyhedral surface in $U$ such that it satisfies condition $\left(\mathrm{C}_{k}\right)$ and $g\left(M_{k}\right)=g(S, U)$.

Step 2. For $i=1, \ldots, m$ and $k=1,2, \ldots$ we know that $M_{k} \cap A_{i k}$ consists of a finite number of simple closed curves in Int $\left(A_{i k}^{\prime}\right)$. Suppose that for fixed $i$ and $k$ there exists a component $L$ of $M_{k} \cap A_{i k}$ such that $L$ bounds a disc $D$ in $A_{i k}^{\prime}$. We wish to show that we may so modify $M_{k}$ as to avoid this situation.

We may assume that $M_{k}$ has no points in common with Int $(D)$. By cutting $M_{k}$ apart along $L$ and attaching two new discs to the cut ends of $M_{k}$, we can manufacture a set $N$ such that $N$ satisfies condition $\left(\mathrm{C}_{k}\right)$ (though $N$ might not be connected), $N \cap A_{i k}=\left(M_{k} \cap A_{i k}\right)-L$, ch $(N)=\operatorname{ch}\left(M_{k}\right)+2$, and $N$ consists of a single 2-manifold or two disjoint 2-manifolds.

Now if $N$ is a single 2-manifold, then $g(N)<g\left(M_{k}\right)=g(S, U)$.

Suppose that $N$ consists of two disjoint 2-manifolds $N_{1}$ and $N_{2}$. We know that $N$ separates $G_{k}$ from $S$ in $\mathrm{Cl}(U)$ and we wish to show that either $N_{1}$ or $N_{2}$ alone does this. Suppose not. There are disjoint $\operatorname{arcs} B_{1}$ and $B_{2}$ such that $B_{i}$ has endpoints $q_{i}$ and $r_{i}$ in $G_{k}$ and $S$ respectively, Int $\left(B_{i}\right) \subset U$, and $B_{i} \cap N_{i}=\varnothing$. There exist arcs $C_{1}$ and $C_{2}$ such that $C_{1}$ goes from $q_{1}$ to $q_{2}$ in $G_{k}$ and $C_{2}$ goes from $r_{1}$ to $r_{2}$ in $S$. Let $J$ be the simple closed curve obtained by taking the union of $B_{1}, B_{2}, C_{1}$, and $C_{2}$. Clearly $B_{2}$ must pierce $N_{1}$ an odd number of times to get from $S$ to $G_{k}$, so the mod 2 intersection number of $J$ with $N_{1}$ is one. But by the way $W$ was chosen, we can homotopy $J$ into $S$ and thus prove that the mod 2 intersection number of $J$ with $N_{1}$ is zero. Contradiction. Thus we may assume that $N_{1}$ separates $G_{k}$ from $S$ in $\mathrm{Cl}(U)$. Hence $N_{1}$ satisfies condition $\left(\mathrm{C}_{k}\right)$. Now using the facts that $\mathrm{ch}(N)$ $=\operatorname{ch}\left(M_{k}\right)+2$, that $N_{1}$ and $M_{k}$ are of the same orientability class, and that $\operatorname{ch}\left(N_{2}\right)$ is less than or equal to 2 , it clearly follows that $g\left(N_{1}\right) \leqq g\left(M_{k}\right)=g(S, U)$.

It is clear from the above description that we can replace each $M_{k}$ by a 2-manifold $P_{k}$ such that $P_{k}$ satisfies condition $\left(\mathrm{C}_{k}\right), g\left(P_{k}\right) \leqq g(S, U)$, and for $i=1, \ldots, m$ each component of the intersection of $A_{i k}$ and $P_{k}$ is a polyhedral simple closed curve which is essential in $A_{i k}$.

Step 3. We should like to show that by a process similar to that of Step 2 we can so modify $P_{k}$ as to reduce the number of components of $P_{k} \cap A_{i k}$ to one and ensure that $P_{k}$ still satisfies condition $\left(\mathrm{C}_{k}\right)$ and has genus not greater than $g(S, U)$. Unfortunately we cannot always do this, but in those cases where we cannot, we 
will be able to show the existence of a disc on $P_{k}$ the boundary of which is one of the components of $P_{k} \cap A_{i k}$.

Let us consider a choice of $k$ and $i$ such that the intersection of $P_{k}$ and $A_{i k}$ contains at least three components. Then there are two such components $F_{1}$ and $F_{2}$ such that $F_{1}$ and $F_{2}$ bound an annulus $A^{*}$ on $A_{i k}$ and Int $\left(A^{*}\right)$ misses $P_{k}$. There exists a solid polyhedral torus $T$ in $U$ such that $A_{i k} \cap \operatorname{Bd}(T)=F_{1} \cup F_{2}, A_{i k} \cap T$ $=A^{*}, T$ is contained in $U-G_{k}, T \cap P_{k}=\mathrm{Bd}(T) \cap P_{k}=$ the union of two disjoint polyhedral annuli $Q_{1}$ and $Q_{2}$, and $F_{j}$ is essential in Int $\left(Q_{j}\right)$ for $j=1,2$.

Let $N=\left(P_{k} \cup \mathrm{Bd}(T)\right)-\left(\operatorname{Int}\left(Q_{1}\right) \cup \operatorname{Int}\left(Q_{2}\right)\right)$. Then $N$ consists of either one or two new 2-manifolds and $\operatorname{ch}(N)=\operatorname{ch}\left(P_{k}\right)$.

Case 1. Suppose $N$ is connected. Then $N$ satisfies condition $\left(\mathrm{C}_{k}\right)$ and $g(N)=$ $g\left(P_{k}\right) \leqq g(S, U)$. The only important difference between $N$ and $P_{k}$ is that $N \cap A_{i k}$ has two components fewer than $P_{k} \cap A_{i k}$.

Case 2. Suppose $N$ consists of two disjoint 2-manifolds, $N_{1}$ and $N_{2}$. From the proof given in Step 2, we see that we can assume that $N_{1}$ separates $G_{k}$ from $S$ in $\mathrm{Cl}(U)$. Clearly $N_{1}$ satisfies condition $\left(\mathrm{C}_{k}\right)$.

Subcase i. Suppose $g\left(N_{2}\right)=0$. We know there is an annulus $A$ on $N_{2}$ such that $N_{2} \cap \mathrm{Bd}(T)=A$ and $N_{2}-A$ is a subset of $P_{k}$. Then there exists a disc $H$ on $N_{2}$ such that $H$ is a subset of $P_{k}$ and $\mathrm{Bd}(H)$ is one of the components of $\mathrm{Bd}(A)$. We can find an annulus $Q^{*}$ such that $Q^{*}$ lies on either $Q_{1}$ or $Q_{2}$ and the boundary components of $Q^{*}$ are $\mathrm{Bd}(H)$ and one of the simple closed curves $F_{1}, F_{2}$. Then $H \cup Q^{*}$ is a disc on $P_{k}$ the boundary of which is an essential simple closed curve on $A_{i k}$.

Subcase ii. Suppose $N_{2}$ is nonorientable and $g\left(N_{2}\right)=1$. Taking $A$ as defined in Subcase i, a few simple genus calculations show that $A$ must separate $N_{2}$ and that one of the components of $N_{2}-$ Int $(A)$ must be a disc $H$. We then go on to the same conclusion as in Subcase i.

Subcase iii. Now consider any possibility other than the two subcases discussed above. Then $\operatorname{ch}\left(N_{2}\right) \leqq 0$ and hence $g\left(N_{1}\right) \leqq g\left(P_{k}\right)$, so that we obtain a situation like that of Case 1.

Now let $F$ be a polyhedral surface in $U$ satisfying condition $\left(\mathrm{C}_{k}\right)$ and consider the following statements:

$F-i: F \cap A_{i k}$ consists of a single simple closed curve which is essential in $A_{i k}$, and $g(F) \leqq g(S, U)$.

$F * i$ : Every component of $F \cap A_{i k}$ is essential in $A_{i k}$, and one of these components bounds a disc on $F$.

It follows from the above considerations that for $k=1,2, \ldots$ we may replace $P_{k}$ with a surface $S_{k}$ satisfying condition $\left(\mathrm{C}_{k}\right)$ such that either $S_{k}-i$ is true for each $i$ or $S_{k} * i$ is true for some $i$.

Step 4. We shall now show that for all but a finite number of $k$ the statement $S_{k}-i$ is true for $i=1, \ldots, m$.

Suppose not. Then for an infinite number of $k$ we know that $S_{k} * i$ is true for 
some $i$. We may as well suppose $S_{k} * 1$ is true for an infinite number of $k$. If $S_{k} * 1$ holds, then there is a disc $D^{\prime}$ on $S_{k}$ such that $\mathrm{Bd}\left(D^{\prime}\right)$ is one of the components of $S_{k} \cap A_{1 k}$. There exists a subdisc $D$ of $D^{\prime}$ such that $\mathrm{Bd}(D)$ is a component of $S_{k} \cap A_{i k}$ for some $i$ (say $i=1$ ) and $A_{j k}$ does not intersect Int (D) for any $j$. By "walking" up the side of $A_{1 k}$, we can construct an $\operatorname{arc} p_{1} a$ such that

$p_{1} a-p_{1} \subset\left(U-A_{1 k}\right) \cap N\left(S, \sigma_{k}\right)$,

$p_{1} a$ intersects $S_{k}$ a finite number of times,

$a$ is a point in $G_{k}$, and

$\operatorname{diam}\left(p_{1} a\right)<\delta_{k}$.

Case 1. Suppose $m \geqq 2$. Then there are arcs $a c, c d$, and $d p_{1}$ such that $a c$ is in $G_{k}$ and does not intersect $S_{k}, d$ is a point of $S, c d \subset A_{2 k}$, and Int $\left(d p_{1}\right) \subset V$.

Let $J$ be the union of $p_{1} a, a c, c d$, and $d p_{1}$ (we may assume $J$ to be a simple closed curve). Now let $A^{*}$ be the subannulus of $A_{1 k}^{\prime}$ bounded by $\operatorname{Bd}\left(D_{1 k}\right)$ and Bd $(D)$, and consider the 2-sphere $S^{\prime}$ obtained by taking the union of $D_{1 k}, A^{*}$, and $D$. We can homotopy $J$ into $S$ (by virtue of our choice of $W$ ) and then into $S-D_{1 k}$. Hence the mod 2 intersection number of $J$ with $S^{\prime}$ is zero. Since $J$ pierces $S^{\prime}$ at $p_{1}$, there must be another point at which $J$ intersects $S^{\prime}$. From our construction it follows that there is a point $b$ in the interior of $p_{1} a$ such that $b$ is a point of $D$. By letting $p_{1} b$ (the obvious subarc of $p_{1} a$ ) take the place of $p a$ in the proof of Lemma 3 , we can show as in that lemma that $\operatorname{diam}(D)<7 \delta_{k}<1 / 2^{k}$. Now let $D_{k}^{*}$ be the disc $D \cup A^{*}$. Since diam $\left(D_{k}^{*}\right)<1 / 2^{k-1}$ and $D_{k}^{*}$ must exist for an infinite number of $k$, it follows that $S$ can be locally spanned from $U$ at $p_{1}$ and hence that $S$ is locally tame from $U$ at $p_{1}[4$, Theorem 10]. But this contradicts the hypotheses of our theorem.

Case 2. Suppose $S$ is not a 2-sphere and $m=1$. There is a simple closed curve $L$ on $S$ such that $L$ does not intersect $p_{1}$ and $L$ is not nullhomotopic in $S$. We can find an annulus $A$ satisfying the requirements that $L$ is one of its boundary components, $A-L$ is contained in $U$, and $A-L$ is locally polyhedral and in general position with respect to $D$. We may assume that we are considering $k$ so large that $A_{1 k}$ and $D_{1 k}$ do not intersect $A$ and $\mathrm{Bd}(A)-L$ is contained in $G_{k}$. We can construct an arc $a c$ so that $a c$ does not intersect $S_{k}$ and $c$ is a point of $A$. Now suppose $D \cap A$ contains a simple closed curve $L^{\prime}$ which is essential in $A$. Let $H=$ the disc on $D$ bounded by $L^{\prime}$ and $B=$ the subannulus of $A$ bounded by $L$ and $L^{\prime}$. Using Lemma 2 and our choice of $W$, we can shove the singular disc $H \cup B$ into $S$ and then use it to show that $L$ is nullhomotopic on $S$. Since this contradicts our choice of $L$, we see that every component of $D \cap A$ must bound a disc in $A$. Hence we can construct an arc $c d$ in $A$ such that $d$ is a point in $S$ and $c d$ does not intersect $D, A_{1 k}$, or $D_{1 k}$. The rest of the proof is a copy of Case 1 .

We therefore conclude that $S_{k} * i$ can be true for at most a finite number of $k$ and $i$.

Step 5. For this last step, we shall consider only values of $k$ for which $S_{k}-i$ is true for $i=1, \ldots, m$. 
We see that $g\left(S_{k}\right) \leqq g(S, U)$ and that since each $S_{k}$ separates $S$ from $G_{k}$ in $\mathrm{Cl}(U)$ we have $\lim S_{k}=S$. For sufficiently large $k$, we see from Lemma 3 that there are pairwise disjoint discs with handles $C_{1 k}, \ldots, C_{m k}$, components of $S_{k}-\operatorname{Int}\left(A_{1 k}\right)$, $\ldots, S_{k}-$ Int $\left(A_{m k}\right)$ respectively, such that $\operatorname{diam}\left(C_{i k}\right)<7 \delta_{k}<1 / 2^{k}$. Let $A_{i k}^{*}=$ the subannulus of $A_{i k}$ between $\mathrm{Bd}\left(C_{i k}\right)$ and $\mathrm{Bd}\left(D_{i k}\right)$. Since $C_{i k} \cup A_{i k}^{*}$ is a disc with $g\left(C_{i k}\right)$ handles which is contained in $U$ except for its boundary, is "cupped" over $p_{i}$, and is of diameter less than $1 / 2^{k-1}$, we then see that we may assume we are working with a value of $k$ for which $g\left(C_{i k}\right)$ is greater than or equal to $g\left(p_{i}, U\right)$. Let $C_{0 k}=\mathrm{Cl}\left(S_{k}-\bigcup_{i=1}^{m} C_{i k}\right)$.

We consider first the case in which $S$ is orientable. We have

$$
g(S, U) \geqq g\left(S_{k}\right)=\sum_{i=0}^{m} g\left(C_{i k}\right) \geqq g\left(C_{0 k}\right)+\sum_{i=1}^{m} g\left(p_{i}, U\right) .
$$

It is now necessary only to show that $g\left(C_{0 k}\right)$ is greater than or equal to $g(S)$. This is trivial if $g(S)=0$, so suppose $g(S) \neq 0$. There exist in $S$ pairwise disjoint discs with one handle $H_{1}, \ldots, H_{N}$ (where $N=g(S)$ ) containing none of the points $p_{1}, \ldots, p_{m}$. Let $H$ be the union of $H_{1}, \ldots, H_{N}$. Since $S$ is locally tame from $U$ at every point of $H$, there is a homeomorphism $h$ from $H \times I$ into $\mathrm{Cl}(U)$ such that $h(x, 0)=x$ and $h(x, t)$ is a point in $U$ for $t>0$ and $x \in H$. We may assume $k$ chosen so large that $h(H \times I)$ intersects $S_{k}$ only in Int $\left(C_{0 k}\right)$ and $S_{k}$ separates $H$ and $h(H \times 1)$ in $\mathrm{Cl}(U)$. Furthermore we may suppose by [1] that $h(H \times(0,1])$ is locally polyhedral and thus may be assumed to be in general position with respect to $C_{0 k}$. It follows then from Lemma 2 of [9] that for $i=1, \ldots, N$ we can find a handle on some component of $C_{0 k} \cap h\left(H_{i} \times I\right)$. These are clearly distinct handles for distinct values of $i$, and thus $g\left(C_{0 k}\right) \geqq g(S)$.

The case in which $S$ is nonorientable is treated in the same way except that $H_{1}, \ldots, H_{N}$ are Moebius strips and one uses Lemma 4 of [9]. The 2 in front of $\sum_{i=1}^{m} g\left(p_{i}, U\right)$ arises from the fact that $g\left(p_{i}, U\right)$ is obtained by counting handles on a disc while $g(S, U)$ is found by computing the genera of nonorientable surfaces approaching $S$. This finishes the proof of the theorem.

4. An application. Daverman in [8] defines a concept, the local enveloping genus of a crumpled cube at a point, which we shall denote $g^{\prime}(p, U)$ and generalize to 2-manifolds in 3-manifolds in the following way:

Definition. Let $S$ be a 2-sided surface in a 3-manifold $M^{3}$ such that $S$ has $U$ for one of its two sides. Let $W^{\prime}$ be the union of $U, S$, and $S \times I$ where every $x$ in $S$ is identified with $(x, 0)$. We know from [11] that $W^{\prime}$ is a 3-manifold at each point of $S$. Choose a point $p$ of $S$ and let $A$ be the $\operatorname{arc} p \times I$. We then define $g^{\prime}(p, U)$ to be the local enveloping genus of $A$ at $p$. Now we need to define local enveloping genus of $A$ at $p$.

Definition. Let $A$ be an arc in $E^{3}$ with endpoints $p$ and $q$. Consider the statement $H(p, n)$ : For every positive real number $\varepsilon$ there is a sphere with $n$ handles $K$ such that $K$ separates $p$ and $q$, the intersection of $K$ and $A$ is a single point in Int $(A)$, 
and $K$ is contained in $N(p, \varepsilon)$. If $H(p, n)$ is true and $H(p, n-1)$ is not, then we define the local enveloping genus of $A$ at $p, \operatorname{LEG}(A, p)$, to be $n$.

Not surprisingly, this turns out to be the same as our $g(p, U)$, and we sketch here a proof of the equivalence of the concepts.

Lemma 5. Let $S$ be a 2-sided surface in a 3-manifold $M^{3}$ such that $S$ has $U$ for one of its sides. Let $p$ be a point in $S$ such that $S$ is locally tame from $U$ at every point except possibly $p$. Then $g(p, U)=g^{\prime}(p, U)$.

Proof. Let $W^{\prime}$ be the union of $U, S$, and $S \times I$ where all the points $x$ in $S$ are identified with $(x, 0)$. Let $A$ be the arc $p \times I$. Then $g^{\prime}(p, U)=\operatorname{LEG}(A, p)$, the local enveloping genus being calculated in the 3-manifold $W^{\prime}$.

Let $H$ be a disc with $g(p, U)$ handles which is "cupped" over $p$ in the manner described in the definition of $g(p, U)$. We can attach a disc $D$ to $H$ in such a way that Int $(D)$ is contained in $S \times(0,1)$, the intersection of $A$ and $D$ consists of a single point, $A$ pierces $D$ at that point, and $\mathrm{Bd}(D)=\mathrm{Bd}(H)$. Since the sphere with $g(p, U)$ handles which is formed by taking the union of $H$ and $D$ can be constructed in an arbitrarily small neighborhood of $p$, we conclude that $g(p, U)$ is greater than or equal to $g^{\prime}(p, U)$.

Now we have to prove that $g(p, U)$ is less than or equal to $g^{\prime}(p, U)$. We see that we can find a sphere with handles $Q$, a simple closed curve $C$, and an annulus $B$ such that $A$ intersects $Q$ in a single point $r$ and pierces it there, $Q$ can be assumed to lie in some arbitrarily small neighborhood of $p$ which has been chosen beforehand, the genus of $Q=g^{\prime}(p, U), B=C \times I \subset S \times I, C \times 0$ bounds a disc $D^{\prime}$ on $S$ such that $p$ lies in Int $\left(D^{\prime}\right), C \times \frac{1}{2}$ bounds a disc $D^{\prime \prime}$ on $Q$ such that $r$ lies in Int $\left(D^{\prime \prime}\right)$, and $B$ intersects $Q$ in precisely the set $C \times \frac{1}{2}$.

Let $Q^{\prime}$ be the disc with $g^{\prime}(p, U)$ handles and boundary $C \times 0$ which is obtained by taking the union of $Q-D^{\prime \prime}$ and $C \times\left[0, \frac{1}{2}\right]$. Since $S-\operatorname{Int}\left(D^{\prime}\right)$ can be collared from $U$ by [3] and that part of $Q^{\prime}$ which intersects $S \times I$ lies in $\left(S-\operatorname{Int}\left(D^{\prime}\right)\right) \times I$, it is not very difficult to construct a homeomorphism $h$ on $Q^{\prime}$ such that $h$ is the identity on $\mathrm{Bd}\left(Q^{\prime}\right)$ and $h\left(\operatorname{Int}\left(Q^{\prime}\right)\right)$ is contained in $U$. Because $h\left(Q^{\prime}\right)$ corresponds to a disc with handles as described in the definition of $g(p, U)$ and $h\left(Q^{\prime}\right)$ can be constructed in an arbitrarily small neighborhood of $p$, it follows that $g^{\prime}(p, U)$ is greater than or equal to $g(p, U)$.

The concept of $g^{\prime}(p, U)$ has the advantage that it can be applied to any 2-manifold embedded in a 3-manifold. Abandoning the temporary notation $g^{\prime}(p, U)$ in favor of the symbol $g(p, U)$, we give the definition of nonpiercing point and generalize Theorem 5 of [8].

Definition. Let $S$ be a 2-sided surface in a 3-manifold $M^{3}$ such that $S$ has $U$ for one of its sides, and let $p$ be a point of $S$. We say that $p$ is a piercing point of $S$ with respect to $U$ if and only if there is a neighborhood $N$ of $p$ in $\mathrm{Cl}(U)$ and a homeomorphism $h$ from $N$ into $E^{3}$ such that $h(S \cap N)$ can be pierced at $p$ by a tame arc. 
THEOREM 2. Let $S$ be a 2-sided surface in a 3-manifold $M^{3}$ such that $S$ has $U$ for one of its sides. Then the number of points of $S$ which are nonpiercing points with respect to $U$ is less than or equal to

1

$$
\text { if } g(S)=g(S, U)=0 \text {, }
$$

$g(S, U)-g(S) \quad$ if $S$ is orientable and either $g(S, U) \neq 0$ or $g(S) \neq 0$, and $\frac{1}{2}(g(S, U)-g(S))$ if $S$ is nonorientable.

Proof. The proof is the same as that of Theorem 5 of [8] except that where Daverman uses [7] and [13], we use Theorem 1 and Lemma 5 of this paper.

\section{REFERENCES}

1. R. H. Bing, Locally tame sets are tame, Ann. of Math. (2) 59 (1954), 145-158. MR 15, 816.

2. - Approximating surfaces with polyhedral ones, Ann. of Math. (2) 65 (1957), 456-483. MR 19, 300.

3. Morton Brown, Locally flat imbeddings of topological manifolds, Ann. of Math. (2) 75 (1962), 331-341. MR 24 \#A3637.

4. C. E. Burgess, Characterizations of tame surfaces in $E^{3}$, Trans. Amer. Math. Soc. 114 (1965), 80-97. MR 31 \#728.

5. - Criteria for a 2-sphere in $S^{3}$ to be tame modulo two points, Michigan Math. J. 14 (1967), 321-330. MR 35 \#7314.

6. J. C. Cantrell, Almost locally polyhedral 2-spheres in $S^{3}$, Duke Math. J. 30 (1963), 249-252. MR $26 \# 5551$.

7. R. J. Daverman, Non-homeomorphic approximations of manifolds with surfaces of bounded genus, Duke Math. J. 37 (1970), 619-625. MR 42 \#2448.

8. - On the number of non-piercing points in certain crumpled cubes, Pacific J. Math. 34 (1970), 33-43.

9. C. H. Edwards, Jr., Concentricity in 3-manifolds, Trans. Amer. Math. Soc. 113 (1964), 406-423. MR 31 \#2716.

10. S. Hu, Theory of retracts, Wayne State Univ. Press, Detroit, Mich., 1965. MR 31 \#6202.

11. L. L. Lininger, Some results on crumpled cubes, Trans. Amer. Math. Soc. 118 (1965), 534-549. MR 31 \#2717.

12. D. R. McMillan, Jr., Neighborhoods of surfaces in 3-manifolds, Michigan Math. J. 14 (1967), 161-170. MR 35 \#3643.

13. M. D. Taylor, An upper bound on the number of wild points on a 2-sphere, $\mathrm{Ph} . \mathrm{D}$. Thesis, Florida State University, Tallahassee, Fla., 1969.

14. R. L. Wilder, Topology of manifolds, 2nd ed., Amer. Math. Soc. Colloq. Publ., vol. 32, Amer. Math. Soc., Providence, R. I., 1963. MR 32 \#440.

Department of Mathematics, Florida Technological University, Orlando, Florida 32816 (Current address of Michael D. Taylor)

Department of Mathematics, University of Alabama, Tuscaloosa, Alabama 35401 (Current address of Harvey Rosen) 